\title{
Bound eigenstates for Yukawa potential through Matrix representation
}

\author{
Soyeon Cho* and Jin-Hee Yoon \\ Department of physics, Inha University \\ Incheon 22212, Repulic of Korea \\ E-mail: jinyoon@inha.ac.kr
}

The Yukawa potential (or screened Coulomb) describes interactions screened by neighbors in many fields of physics: solid state, atomic, nuclear and etc. Especially, in high energy physics, the Yukawa potential is used for the phenomenological quark-quark interaction which, however, needs to be solved numerically since it has no analytic solution. In this work, we try to find the efficient way of the numerical method based on basic principles of Qunatum mechanics; particle states can be expanded with any complete set of eigenstates. We test the validity of this method by solving the Schrödinger equation for the Coulomb potential which has analytic solutions, and we abstract serveral factors affecting the accuracy of solutions: the resemblance between the reference potential and the potential in question, the number of eigenbases and the range of principal quantum numbers of eigenbases.

To obtain eigenenergies and eigenstates for the Yukawa potential, we choose the Coulomb potential as the reference potential and its eigenstates from ground to 100th states. We compare our results to other published ones: Direct root finding method (Direct) [1], Ritz method with Dirichelet boundary condition (Ritz) [2], Super Symmetric quantum mechanics perturbation method (SUSY) [3], Asymptotic Iteration Method (AIM) [4] and Nikiforov-Uvarov method (NU) [5]. The relative errors for eigenenergies are within $2.2 \%$ and those for expectation values of the position are within $0.075 \%$. Also we check that the stronger screening effect makes eigenenergies deeper and wavefunctions wider. This effect is more significant for higher states.

ICHEP2018, XXXIX International Conference on High Energy Physics

4-11 July 2018

Seoul, Republic of Korea

${ }^{*}$ Speaker. 

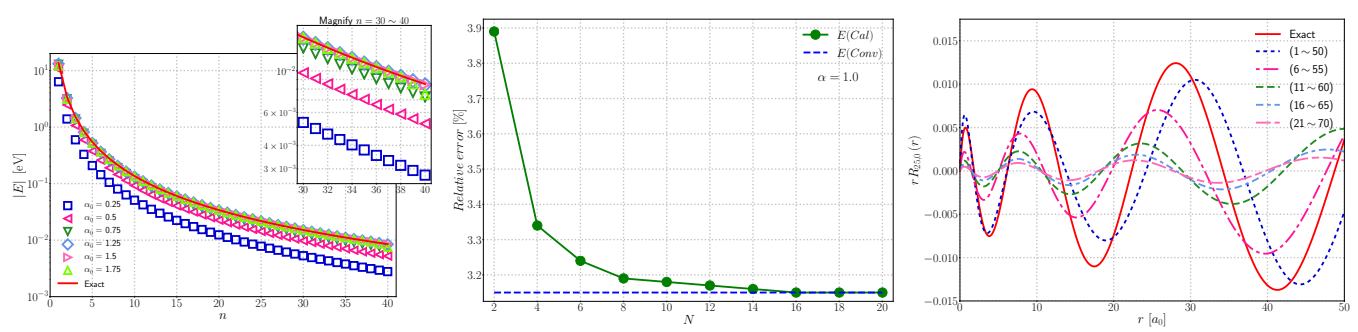

Figure 1: We found important factors affecting the accuracy; the reference potential should be chosen to resemble the target potential as much as possible (Left), the number of eigenbases should be large enough but have no significant effect after the certain value (Middle) and the range of bases should start from the ground state (Right).
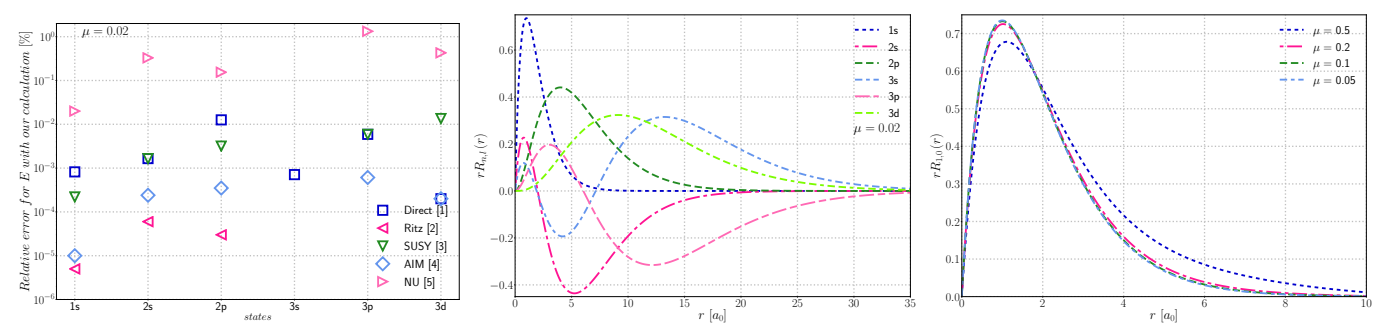

Figure 2: Calculational results: relative errors for eigenenergies with references [1, 2, 3, 4, 5] (Left), $1 s \sim 3 d$ state wavefunctions for $\mu=0.02$ (Middle) and groun state wavefunctions for $\mu=0.05,0.1,0.2,0.5$ (Right).
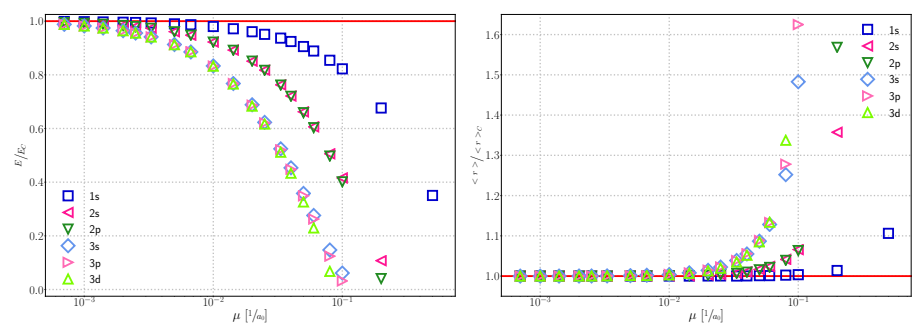

Figure 3: As the screening parameter is increasing, eigenenergies become smaller (Left) and expectation values of the position become larger (Right). Such behavior is more significant at higher states.

\section{References}

[1] F. J. Rogers, H. C. G. Jr and D. J. Harwood, Bound eigenstates of the static screened Coulomb potential, Physical Review A 1 (1970) 1577.

[2] M. A. Núñez, Accurate computation of eigenfunctions for Schrödinger operators associated with Coulomb-type potentials, Physical Review A 47 (1993) 3620.

[3] B. Gönül, K. Köksal and E. Bakir, An alternative treatment for Yukawa-type potentials, Physica Scripta 73 (2006) 279.

[4] M. Karakoc and I. Boztosun, Accurate iterative and perturbative solutions of the Yukawa potential, International Journal of Modern Physics E 15 (2006) 1253.

[5] M. Hamzavi, M. Movahedi, K.-E. Thylwe and A. A. Rajabi, Approximate analytical solution of the Yukawa potential with arbitrary angular momenta, Chinese Physics Letters 29 (2012) 80302. 